\title{
Uso de rastreadores para detecção de eventos adversos aos medicamentos em hospital universitário
}

\author{
Use of triggers tool to identify adverse drug events in an university hospital
}

Leticia dos Santos Sanchesa, Fabíola Giordanib , Jakeline Liara Telekenc, Ana Flávia Gallas Leivas ${ }^{d}$,Raysa Cristina Schmidtd, Jessica Cristina Balbinotc, Anna Paula Moreira Garbuio ${ }^{c}$, Simone Cavalli Pianna ${ }^{d}$

a Farmacêutica. Residência em Farmácia Hospitalar, Universidade Estadual do Oeste do Paraná.

${ }^{\mathrm{b}}$ Farmacêutica. Doutora em Saúde Pública, Universidade Federal Fluminense.

c Farmacêutica. Universidade Estadual do Oeste do Paraná.

¿ Médica. Universidade Estadual do Oeste do Paraná.

Fontes de financiamento: Bolsa de residência com recursos Secretaria da Ciência, Tecnologia e Ensino Superior - Estado do Paraná para LSS.

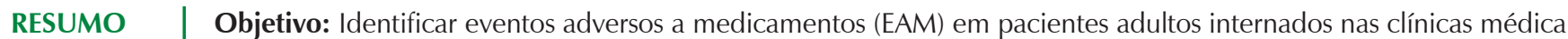
e cirúrgica em um hospital universitário, bem como caracterizar esses eventos.

Materiais e Métodos: Foi realizado estudo retrospectivo em um hospital universitário. A amostra foi constituída pelos prontuários dos pacientes internados nas clínicas médica e cirúrgica entre outubro de 2010 e janeiro de 2011. Para identificação dos EAM aplicou-se um conjunto de rastreadores que são condições que podem indicar que um EAM ocorreu e tornam a revisão do prontuário mais objetiva e direcionada. Esses rastreadores podem ser um exame laboratorial alterado, o uso de um antídoto, ou uma condição clínica.

Resultados: O grupo de estudo foi constituído de 263 pacientes, dos quais $58(22,1 \%)$ tiveram ao menos um EAM durante a internação e 83 EAM foram identificados, resultando em uma taxa de 31,5 EAM por 100 pacientes. Os eventos mais frequentes foram náusea e vômito, erupções cutâneas e prurido e hipocalemia. Quanto aos medicamentos imputados o tramadol foi o mais frequentemente relacionado aos eventos. Os eventos foram mais presentes em pacientes com internação com caráter de urgência/emergência, que possuíam uma ou mais comorbidades. Ainda, pacientes com eventos apresentaram um tempo médio de internação mais prolongado.

Conclusão: O uso de rastreadores para identificar EAM apresenta-se como estratégia útil, com a qual foi possível identificar que 22,1\% dos pacientes apresentaram EAM durante a internação. Tal estratégia de identificação de eventos é passível de ser implementada pelos núcleos de segurança do paciente de instituições hospitalares.

Palavras-chave: sistemas de notificação de reações adversas a medicamentos; monitoramento de medicamentos; hospitais; hospitalização.

ABSTRACT
Objective: To identify adverse drug events (ADE) in adults patients hospitalized in medical and surgical units of a university hospital, as well as to characterize these events.
Materials and Methods: A retrospective study was performed in a university hospital. The sample consisted of patients admitted to the medical and surgical units between October 1st, 2010 and January 315t, 2011. In order to identify the ADE, a set of triggers was applied, which are conditions that may indicate that an ADE occurred and make the review of records more objective and direct. These triggers can be an altered laboratory test, the use of an antidote or a clinical condition.
Results: The study group consisted of two hundred and sixty-three patients, of whom fifty-eight (22.1\%) had at least one ADE during hospitalization and 83 ADE were identified, resulting in a rate of 31.5 ADE per 100 patients. The most common events were nausea and vomiting, rash and itching and hypokalemia. As for the alleged drug, tramadol was most often related to the events. The events were more present in patients with urgent/emergency hospitalization, that had one or more comorbidities. Also, patients with ADE had a longer average time of hospitalization.
Conclusion: The use of triggers to identify ADE presents as a useful strategy, from which it was possible to identify that $22.1 \%$ of patients had an ADE during hospitalization. This strategy to identify the events is likely to be implemented by patient safety core hospitals.
Keywords: adverse drug reaction reporting systems; drug monitoring; hospitals; hospitalization.

\section{Correspondência:}




\section{INTRODUÇÃO}

O uso de medicamentos é uma estratégia terapêutica utilizada para a profilaxia, diagnóstico e tratamento de doenças. Apesar de apresentarem benefícios, os medicamentos são capazes de causar eventos adversos, que são quaisquer danos, graves ou leves, causados por um medicamento empregado com finalidade terapêutica ${ }^{1}$. Estudos demonstram que este é um problema frequente que afeta pacientes hospitalizados e ambulatoriais ${ }^{2,3,4}$. Recente revisão sistemática da literatura mostrou que a proporção de pacientes com Eventos Adversos aos Medicamentos (EAM) durante a internação hospitalar varia de 1,7 a 15,6\%, sendo que uma proporção considerável destes eventos poderia ser evitada ${ }^{4}$.

Diversas abordagens têm sido utilizadas para identificar e quantificar EAM, entre os métodos mais convencionais estão à notificação voluntária, estudos de revisão retrospectiva ou prospectiva de prontuários ${ }^{5}$, e busca de eventos em bases de dados administrativas através de lista de códigos da Classificação Internacional de Doença (CID), na sua nona (CID-9) ou décima (CID-10) versões ${ }^{6,7}$. A notificação voluntária é um método limitado devido à subnotificação, não permitindo avaliar o impacto real dos $\mathrm{EAM}^{8,9}$ e apenas um em cada vinte eventos ocorridos são detectados por este método ${ }^{10}$. O uso de códigos da Classificação Internacional de Doença aplicados a base de dados administrativas, embora permita a identificação de estimativas de abrangência nacional, também é vulnerável a subnotificação ${ }^{7}$. Os demais demandam tempo e recursos humanos em excesso ${ }^{11}$. No final de 2003 o Institute for Healthcare Improvement (IHI) publicou um método alternativo à revisão retrospectiva de prontuários, o qual consiste de um conjunto de 19 rastreadores, incluindo parâmetros laboratoriais, medicamentos utilizados pelo paciente e registros de cuidado e evolução clínica do paciente, que atuam como alertas, e quando encontrados requerem novas investigações para determinar a ocorrência ou não de $\mathrm{EAM}^{12}$. Esta técnica torna a revisão de prontuários mais objetiva, direcionada e com necessidade de menor tempo para sua realização.

Publicações recentes têm descrito a aplicação e uso de rastreadores na identificação da incidência e caracterização dos EAM durante o processo de cuidado no ambiente hospitalar ${ }^{13-16}$. O conhecimento da magnitude em que os eventos ocorrem é etapa necessária para o desenvolvimento de estratégias para ampliar a segurança do paciente. Neste contexto, o presente estudo teve como objetivo identificar EAM em pacientes adultos internados na clínica médica e cirúrgica em um hospital universitário, bem como caracterizar esses eventos.

\section{MATERIAIS E MÉTODOS}

Estudo de coorte retrospectiva com revisão de prontuários, realizado em um hospital universitário usando aplicação de rastreadores para identificação de EAM. Foram utilizados os rastreadores propostos pelo $\mathrm{IHI}$ acrescidos de um conjunto de rastreadores para os quais foi avaliada sua aplicabilidade no contexto de um hospital universitário. O projeto foi aprovado pelo Comitê de Ética em Pesquisa, por meio do parecer 039/2009 - CEP e não apresentou conflitos de interesse.

\section{Definição de EAM}

Evento adverso a medicamento é qualquer dano que ocorra durante o tratamento medicamentoso, seja pelo seu uso adequado ou não'1.

\section{Caracterização e seleção de amostra}

O estudo foi realizado em um hospital universitário com 195 leitos para cuidados de nível secundário e terciário a pacientes do Sistema Único de Saúde (SUS), com diversas especialidades clínicas e cirúrgicas. A amostra foi constituída por pacientes admitidos na unidade de clínica médica e cirúrgica entre 01 de outubro de 2010 e 31 de janeiro de 2011. Os registros das internações dos meses estudados foram extraídos a partir dos bancos de dados que alimentam o Sistema de Informação Hospitalar (SIH-SUS). Os prontuários dos pacientes incluídos foram revisados dia a dia por todo período de hospitalização até a alta. Pacientes internados por menos de 48 horas foram excluídos do estudo. Excluíram-se também os dias de hospitalização em unidades de terapia intensiva.

\section{Coleta de dados e variáveis do estudo}

A equipe para a coleta dos dados foi constituída por seis acadêmicos do quarto e quinto ano dos cursos de farmácia e medicina. Os estudantes foram treinados para realizar a extração dos dados. Utilizou-se instrumento padronizado acompanhado de manual com as instruções de busca e preenchimento, definições importantes e lista de rastreadores. A coleta de dados foi realizada de dezembro de 2011 a março de 2012.

Foram extraídas variáveis demográficas e sociais, e dados relacionadas à saúde e a internação do paciente. Entre as variáveis demográficas e sociais estão: gênero, raça/cor da pele e data de nascimento. Já entre os dados relacionados à saúde e à internação do paciente pesquisou-se: duração da internação, caráter da internação (urgência/emergência ou internação eletiva), tipo de atendimento (clínico, cirúrgico ou ambos), resultado do cuidado (alta hospitalar, transferência ou óbito), comorbidades presentes na admissão do paciente, necessidade de internação em unidade de terapia intensiva. 
A lista de rastreadores do $\mathrm{IHI}^{12}$ foi ajustada para adaptação dos mesmos à realidade do hospital estudado. A estes rastreadores foram acrescentados "Elevação da aspartato aminotransferase (AST)", "Elevação da alanina aminotransferase (ALT)", "Elevação de amilase", "Elevação de lipase" e "Alteração de potássio". Os quatro primeiros foram considerados rastreadores positivos quando o valor de uma aferição consecutiva dobrou em relação à anterior. Para o rastreador "alteração de potássio" considerou-se rastreador positivo alteração de $0,5 \mathrm{mg} / \mathrm{dL}$ abaixo ou acima do valor de referência utilizado pelo hospital. O uso desses rastreadores foi incorporado com base em revisão da literatura a qual os apresentou como capazes de identificar eventos relacionados ao uso de medicamentos ${ }^{17}$.

Os medicamentos prescritos durante o período estudado foram registrados em Ficha para Coleta de Medicamentos ${ }^{16}$. Nesta ficha pôde ser acompanhado o período no qual o medicamento permaneceu prescrito e os dias em que foi utilizado.

A busca de rastreadores e a identificação de EAM foram realizadas por dois revisores, de forma independente, seguindo a ordem recomendada pelo $\mathrm{IHI}^{12}$. Primeiramente realizouse a busca de rastreadores em laudos de exames laboratoriais presentes no prontuário eletrônico. Procedeu-se então a busca de rastreadores relacionados à medicação em Fichas de Coleta de Medicamentos. Por último, revisaram-se em prontuário manual todos os registros das equipes médicas e de enfermagem, destacando rastreadores encontrados e relatos que sugerissem EAM. Foi considerada indicação de EAM o registro em prontuário de um evento como descrito, ou intercorrências que pudessem ser associadas ao uso de medicamentos.

Os prontuários com divergências entre as buscas de rastreadores e identificação de EAM foram reavaliados por um farmacêutico residente. Nos casos em que persistiu alguma dúvida quanto à possibilidade de um EAM ter acontecido, um segundo profissional de saúde foi consultado.

\section{Análise dos dados}

Os dados coletados nas diversas etapas foram digitalizados usando o programa Epidata 3.0. O SPSS 17 foi usado para a análise dos dados. A frequência de pacientes com e sem EAM foi calculada para cada variável demográfica e social, e para cada um dos dados relacionados à saúde e a internação do paciente. O teste do qui-quadrado e o teste de t de Student foram realizados para comparar as características das internações e dos pacientes com e sem presença de EAM. A incidência de pacientes com EAM foi estimada (número de pacientes com EAM/número de pacientes incluídos no estudo), bem como a taxa de EAM por 100 pacientes (número de EAM identificados/número de pacientes incluídos no estudo x 100).
O desempenho dos rastreadores e a sua capacidade de captar eventos adversos aos medicamentos foram analisados através da taxa de ocorrência do rastreador, calculada dividindo-se o número de registros de cada rastreador pelo total de prontuários avaliados, multiplicado por 100; pela taxa de ocorrência de EAM, calculada pela divisão do número de EAM identificados pelos rastreadores pelo total de prontuários avaliados, multiplicado por 100; e pelo chamado valor preditivo positivo (VPP) dos rastreadores, calculado dividindo o número de rastreadores que identificaram um EAM pelo total de rastreadores identificados apresentado em porcentagem. Este último é uma proporção que define o rendimento do rastreador e expressa, em valores relativos, o potencial de cada um deles para identificar EAM.

\section{RESULTADOS}

Duzentos e setenta e dois pacientes foram internados no setor de clínica médica e cirúrgica do hospital no período estudado. Após submissão aos critérios de exclusão, nove pacientes foram excluídos por permanecerem hospitalizados por um período inferior a 48 horas. Entre os 263 pacientes incluídos, a maioria era do sexo feminino $(54,4 \%)$, brancos $(84,4 \%)$ e com idade média de $47,2 \pm 20,0$ anos. A maioria das internações teve caráter de urgência ou emergência $(74,9 \%)$, com predomínio de atendimento do tipo cirúrgico $(52,5 \%)$ (Tabela 1) com tempo mediano de internação de oito dias, variando de dois a 95 dias.

Cinquenta e oito pacientes apresentaram pelo menos um EAM durante a internação, o que levou a incidência de 22,1\%, sendo 83 EAM distintos identificados, resultando em uma taxa de 31,5 EAM por 100 pacientes. A presença de pelo menos uma comorbidade $(p=0,011)$, internações de urgência/emergência $(p<0,001)$ e maior tempo de internação $(p=0,001)$ apresentaram associação com a ocorrência de EAM.

A Tabela 2 apresenta os EAM observados, o número de vezes que ocorreram e os medicamentos imputados. Os eventos mais frequentes foram náusea e vômito (21), afecções cutâneas (14) e hipocalemia (8). Entre as classes de medicamentos imputados aos eventos a mais frequente foi a dos analgésicos opióides, sendo o tramadol relacionado a 12 eventos.

Os rastreadores foram identificados em $89,9 \%$ dos prontuários analisados, num total de 737 , com média de 2,5 rastreadores por prontuário, podendo chegar a 17 rastreadores por prontuário. Em 136 (18,4\%) das vezes que um rastreador foi identificado, ele colaborou com a identificação de um dos 83 EAM, sendo que em muitos casos, mais do que um rastreador identificou um mesmo evento, por exemplo, no caso de um evento de hipoglicemia, o paciente apresentou-se letárgico e sonolento, com uma glicemia de $39 \mathrm{mg} / \mathrm{dL}$ e a insulina foi suspensa. 
Tabela 1. Características dos pacientes, segundo ocorrência de eventos adversos a medicamentos (EAM), identificados por rastreadores em hospital Universitário.

\begin{tabular}{|c|c|c|c|c|}
\hline Característica dos pacientes/internações & Sem EAM (\%) & Com EAM (\%) & Total (\%) & Valor de $p$ \\
\hline $\begin{array}{l}\text { Sexo } \\
\text { Feminino } \\
\text { Masculino }\end{array}$ & $\begin{array}{c}112(78,3) \\
93(77,5)\end{array}$ & $\begin{array}{l}31(21,7) \\
27(22,5)\end{array}$ & $\begin{array}{l}143(54,4) \\
120(45,6)\end{array}$ & 0,495 \\
\hline Idade média em anos (DP) & $46,8 \pm 19,8$ & $48,7 \pm 20,9$ & $47,2 \pm 20,0$ & 0,547 \\
\hline $\begin{array}{l}\text { Raça/cor de pele } \\
\text { Branco } \\
\text { Pardo } \\
\text { Negro } \\
\text { Sem informação }\end{array}$ & $\begin{array}{c}171(77,0) \\
28(84,8) \\
2(66,7) \\
4(80,0)\end{array}$ & $\begin{array}{l}51(23,0) \\
5(15,2) \\
1(33,3) \\
1(20,0)\end{array}$ & $\begin{array}{c}222(84,4) \\
33(12,5) \\
3(1,1) \\
5(1,9)\end{array}$ & 0,739 \\
\hline $\begin{array}{l}\text { Caráter da internação } \\
\text { Urgência/Emergência } \\
\text { Eletivo }\end{array}$ & $\begin{array}{c}144(73,1) \\
61(92,4)\end{array}$ & $\begin{array}{c}53(26,9) \\
5(7,6)\end{array}$ & $\begin{array}{c}197(74,9) \\
66(25,1)\end{array}$ & $<0,001$ \\
\hline $\begin{array}{l}\text { Tipo de atendimento } \\
\text { Cirúrgico } \\
\text { Clínico } \\
\text { Cirúrgico e clínico }\end{array}$ & $\begin{array}{l}113(81,9) \\
79(73,8) \\
13(72,2)\end{array}$ & $\begin{array}{c}25(18,1) \\
28(26,2) \\
5(27,8)\end{array}$ & $\begin{array}{c}138(52,5) \\
107(40,7) \\
18(6,8)\end{array}$ & 0,267 \\
\hline Tempo médio de internação em dias (DP) & $11,4(13,5)$ & $18,2(14,0)$ & $12,9(13,9)$ & 0,001 \\
\hline $\begin{array}{l}\text { Necessitou internação em UTI } \\
\text { Não } \\
\text { Sim }\end{array}$ & $\begin{array}{c}172(78,9) \\
33(73,3)\end{array}$ & $\begin{array}{l}46(21,1) \\
12(26,7)\end{array}$ & $\begin{array}{c}218(82,9) \\
45(17,1)\end{array}$ & 0,262 \\
\hline $\begin{array}{l}\text { Comorbidades presentes } \\
\text { Não } \\
\text { Sim }\end{array}$ & $\begin{array}{c}85(85,0) \\
120(73,2)\end{array}$ & $\begin{array}{l}14(14,1) \\
44(26,1)\end{array}$ & $\begin{array}{c}99(37,6) \\
164(62,4)\end{array}$ & 0,011 \\
\hline $\begin{array}{l}\text { Situação de alta } \\
\text { Melhora } \\
\text { Óbito }\end{array}$ & $\begin{array}{c}192(78,7) \\
13(68,4) \\
\end{array}$ & $\begin{array}{c}52(21,3) \\
6(31,6) \\
\end{array}$ & $\begin{array}{c}244(92,8) \\
19(7,2)\end{array}$ & 0,220 \\
\hline
\end{tabular}

Tabela 2. Eventos adversos a medicamentos (EAM) e medicamentos imputados em um hospital Universitário.

\begin{tabular}{|c|c|c|}
\hline EAM & $\mathbf{n}$ & Medicamentos imputados \\
\hline Náusea e vômito & 21 & $\begin{array}{l}\text { Tramadol, nalbufina, ranitidina, morfina, tramadol+morfina, antiretrovirais, } \\
\text { polimixina B, dipirona, cefazolina }\end{array}$ \\
\hline Erupção cutânea e prurido & 14 & $\begin{array}{l}\text { Clindamicina, ceftriaxona + ranitidina, piperacilina +tazobactam, tramadol, } \\
\text { vancomicina, cetoprofeno, ciprofloxacina, nalbufina, ranitidina, contraste iodado, } \\
\text { dipirona +ampicilina, zidovudina, lamivudina }\end{array}$ \\
\hline Hipocalemia & 8 & $\begin{array}{l}\text { Hidroclorotiazida, espironolactona, furosemida, fenoterol, piperacilina+tazobactam, } \\
\text { antimoniato de N-metilglucamina }\end{array}$ \\
\hline Sonolência ou sedação & 5 & Levomepromazina, diazepam, haloperidol, morfina, tramadol \\
\hline Elevação da creatinina & 4 & $\begin{array}{l}\text { Antimoniato de } \mathrm{N} \text {-metilglucamina, pirazinamida + isoniazida + rifampicina + etambutol, } \\
\text { cetoprofeno, ciclofosfamida }\end{array}$ \\
\hline Hipotensão & 4 & $\begin{array}{l}\text { Clonidina + propranolol+captopril+hidroclorotiazida, captopril+furosemida, } \\
\text { captopril, antihipertensivos }\end{array}$ \\
\hline Bradicardia & 2 & Digoxina \\
\hline Elevação de amilase & 2 & Ceftriaxona \\
\hline Elevação da aspartato transaminase & 2 & Clindamicina, furosemida \\
\hline Elevação de lipase & 2 & Ceftriaxona \\
\hline Diarreia & 2 & Ampicilina + gentamicina + metronidazol, vancomicina \\
\hline Hipoglicemia & 2 & Insulina \\
\hline Sangramento & 2 & Heparina \\
\hline Tosse seca & 2 & Captopril \\
\hline Tempo parcial de tromboplastina $>100$ segundos & 2 & Varfarina, heparina \\
\hline Relação Normatizada Internacional > 6 & 1 & Varfarina \\
\hline Leucopenia & 1 & Furosemida \\
\hline Hipercalemia & 1 & Piperacilina e Tazobactam \\
\hline Dor e tremores & 1 & Ciclofosfamida \\
\hline Mielotoxicidade & 1 & Sulfadiazina \\
\hline Neutropenia & 1 & Captopril \\
\hline Pancreatite & 1 & Antimoniato de $\mathrm{N}$-metilglucamina \\
\hline Petéquias & 1 & Heparina \\
\hline Tontura & 1 & Nalbufina \\
\hline
\end{tabular}


Tabela 3. Frequência de rastreadores encontrados, de eventos adversos a medicamentos identificados e valor preditivo positivo em um hospital Universitário.

\begin{tabular}{|c|c|c|c|}
\hline Lista de rastreadores & R por 100 prontuários & EAM por 100 pacientes & VPP* \\
\hline Antialérgicos (dexclorfeniramina, epinefrina, loratadina) & 9,1 & 3,8 & 41,7 \\
\hline Coagulantes (vitamina K e protamina) & 0,4 & 0,0 & 0,0 \\
\hline Antagonista benzodiazepínico & 0,0 & 0,0 & - \\
\hline Antieméticos (bromoprida, metoclopramida e ondansetrona) & 64,6 & 7,6 & 11,8 \\
\hline Antagonista de opióide & 0,0 & 0,0 & - \\
\hline Antidiarreicos (loperamida) & 0,4 & 0,0 & 0,0 \\
\hline Resina de troca iônica (poliestirenossulfonato de cálcio) & 4,2 & 1,1 & 27,3 \\
\hline $\begin{array}{l}\text { Uso de digoxina associado à arritmia, bradicardia, náuseas, vômitos, } \\
\text { anorexia ou alterações visuais }\end{array}$ & 0,8 & 0,8 & 100,0 \\
\hline Interrupção abrupta da medicação & 80,2 & 17,5 & 21,8 \\
\hline Glicemia $<50 \mathrm{mg} / \mathrm{dL}$ & 4,6 & 0,4 & 8,3 \\
\hline Exame positivo para C. difficile nas fezes & 0,0 & 0,0 & - \\
\hline Tempo parcial de tromboplastina (TPP) $>100$ segundos & 4,2 & 1,1 & 27,3 \\
\hline Relação Normatizada Internacional (INR) $>6$ & 1,5 & 0,4 & 25,0 \\
\hline Leucócitos (CGB) <3000 células/mm³ & 8,7 & 1,1 & 13,0 \\
\hline Plaquetas $<50000$ plaquetas $/ \mathrm{mm}^{3}$ & 4,6 & 0,0 & 0,0 \\
\hline Elevação da creatinina sérica & 15,2 & 2,3 & 15,0 \\
\hline Elevação da aspartato transaminase (AST) & 3,8 & 0,8 & 20,0 \\
\hline Elevação da alanina transaminase (ALT) & 1,9 & 0,0 & 0,0 \\
\hline Alteração de potássio & 18,3 & 3,0 & 17,0 \\
\hline Elevação de amilase & 3,8 & 1,1 & 30,0 \\
\hline Elevação de lipase & 3,8 & 1,1 & 30,0 \\
\hline Sedação excessiva, letargia, queda, hipotensão, sonolência ou torpor & 15,6 & 4,6 & 29,3 \\
\hline Rash cutâneo & 3,8 & 3,4 & 90,0 \\
\hline Transferência para nível de cuidado mais complexo & 1,9 & 0,0 & 0,0 \\
\hline
\end{tabular}

* VPP: valor preditivo positivo.

Entre os 24 rastreadores aplicados, três ("Antagonistas de benzodiazepínicos", "Antagonistas de opióides" e "Exame positivo para Clostridium difficile nas fezes") não foram identificados em nenhum dos prontuários analisados. Outros cinco ("Coagulantes", "Antidiarréicos", "Plaquetas < 50000 plaquetas $/ \mathrm{mm}^{3}$ ", "Elevação da alanina aminotransferase (ALT)" e "Transferência para UTI"), embora presentes nos prontuários, não identificaram EAM. Os rastreadores identificados com maior frequência foram "Interrupção abrupta da medicação", presente em 80,2\% dos prontuários, e "Antieméticos", presentes em $64,6 \%$ dos prontuários. O VPP de cada rastreador variou de 8,3 a $100 \%$, sendo que os rastreadores que identificaram maior número de eventos foram "Uso de digoxina associado à arritmia, bradicardia, náuseas, vômitos, anorexia ou alterações visuais" (100\%), "Rash cutâneo" (90\%), "Antialérgicos" (41,7\%), "Relação Normatizada Internacional (INR) > 6" (40\%) e "Elevação de amilase e lipase" (30\%).

\section{DISCUSSÃO}

No presente estudo, a cada 100 pacientes internados cerca de 31 apresentaram EAM, estimativa semelhante à encontrada por Tribiño et al. ${ }^{18}$ em um hospital universitário da Colombia onde a taxa encontrada foi de 32 EAM por 100 pacientes.

Estudos que utilizaram a metodologia de rastreadores para captura de EAM encontraram resultados variados como $1,73 \%{ }^{19}, 5,3 \%^{20}, 19,2 \%^{21}$ e $31,3 \%{ }^{22}$ dos pacientes com EAM. No presente estudo, estimou-se que $22,1 \%$ dos pacientes hospitalizados apresentaram ao menos um EAM. Comparar as taxas de EAM entre distintos hospitais é algo desafiador, uma vez que as estimativas podem ser afetadas por inúmeras variáveis, tais como distintas técnicas de identificação dos eventos, o perfil dos pacientes estudados e características dos serviços. Além do mais, determinar a ocorrência de eventos adversos em ambientes hospitalares é uma tarefa complexa, 
o evento pode confundir-se à doença, e pode ser causado por diversos fatores, incluindo respostas específicas de cada paciente, perfil farmacocinético do medicamento, ou erro de medicação $0^{23}$.

Estudos indicam aumento no tempo de internação dos pacientes que desenvolveram EAM em comparação aos que não apresentaram eventos ${ }^{24,25}$, o que corrobora com os resultados aqui apresentados. Quanto ao tipo de internação, às com caráter de urgência/emergência tiveram maior probabilidade de apresentar um evento em relação às internações eletivas, assim como os pacientes que apresentaram ao menos uma comorbidade em relação aos que não tinham comorbidades relatadas. As diferenças levantam a hipótese de que pacientes com quadro clínico de maior complexidade tinham maior probabilidade de apresentarem complicações no cuidado e maior propensão a desenvolverem EAM, entretanto esta associação precisa de análises complementares para ser melhor entendida.

No presente estudo encontramos VPP total de $18,4 \%$. O VPPé uma medida que demonstra o potencial dos rastreadores em captar eventos. Os rastreadores que apresentaram melhor rendimento em identificar eventos foram "Uso de digoxina associado com sintomas de intoxicação", "Rash cutâneo", "Antialérgicos" e "Relação normatizada internacional", sendo que estes não foram os mais encontrados, fato que representa menor carga de trabalho. Quanto maior número de rastreadores por 100 prontuários, maior será a carga de trabalho para identificação dos EAM.

Os rastreadores aqui incorporados ("Elevação da aspartato aminotransferase (AST)", "Elevação da alanina aminotransferase (ALT)", "Elevação de amilase", "Elevação de lipase" e "Alteração de potássio") à lista do IHI apresentaramse úteis na identificação de eventos, contribuindo com a identificação de 16 eventos, representando 19\% dos EAM identificados. A capacidade de cada um destes rastreadores em identificar eventos mostrou-se compatível com resultados de estudos internacionais ${ }^{17}$.

Uma das limitações do estudo foi seu caráter retrospectivo. Sabe-se que neste tipo de estudo conta-se com as informações disponíveis, e muitas vezes estas informações estão incompletas. A falta de registro dos acontecimentos diários na evolução do paciente também dificulta a caracterização dos EAM. Ter excluído os dias de internamento em UTI também pode ter limitado a identificação de EAM, já que reações tardias podem acontecer.

Um atributo que colaborou para o processo de captação de EAM foi a utilização da Ficha para Coleta de Medicamentos ${ }^{16}$. Esta permitiu visualizar e acompanhar a evolução do tratamento medicamentoso ao longo do tempo, além de relacionar os medicamentos com os parâmetros laboratoriais e evolução do paciente. Os resultados dos exames laboratoriais estavam disponíveis em sistema informatizado, o que também contribuiu para a captação de rastreadores, proporcionando mais agilidade na coleta de dados. Outro ponto positivo foi realizar todas as etapas de extração de dados e análise de EAM e rastreadores em duplicata, pois permitiu complementar dados que passaram despercebidos por um único investigador.

Os resultados aqui apresentados corroboram a evidência da importância de realizar o diagnóstico dos EAM em pacientes hospitalizados. O melhor entendimento dos aspectos a eles relacionados torna possível fomentar a formulação de políticas de segurança do paciente quanto ao uso de medicamentos. $\mathrm{O}$ uso de rastreadores para identificar EAM apresenta-se como estratégia útil, passível de ser implementada pelos núcleos de segurança do paciente de instituições hospitalares.

\section{REFERÊNCIAS}

1. SP-SQS - Committee of Experts on Management of Safety and Quality in Health Care. Expert group on safe medication practices. Glossary of terms related to patient and medication safety [Internet]. WHO; 2005 [cited 2016 Jun 21]. Available from: http://www.who. int/patientsafety/highlights/COE_patient_and_medication_safety gl.pdf

2. Kohn LT, Corrigan JM, Donaldson MS. To Err Is Human: building a safer health system. Washington, DC: IOM; 2000.

3. Pirmohamed M, James S, Meakin S, Green C, Scott AK, Walley TJ, Farrar K, Park BK, Breckenridge AM. Adverse drug reactions as cause of admission to hospital: prospective analysis of 18820 patients. BMJ. 2004;329(7456):15-9. http://dx.doi.org/10.1136/ bmj.329.7456.15

4. Martins AC, Giordani F, Rozenfeld S. Adverse drug events among adult inpatients: a meta-analysis of observational studies. J Clin Pharm Ther. 2014;39(6):609-20. http://dx.doi.org/10.1111/ jcpt.12204

5. Griffin FA, Resar RK. IHI Global Trigger Tool for Measuring Adverse Events [Internet]. Cambridge: Institute for Healthcare Improvement; 2009. [cited 2016 Jun 21]. Available from: http://www.ihi.org

6. Hougland P, Xu W, Pickard S, Masheter C, Williams SD. Performance of International Classification Of Diseases, 9th Revision, Clinical Modification codes as an adverse drug event surveillance system. Med Care. 2006;44(7):629-36. http://dx.doi.org/10.1097/01. mlr.0000215859.06051.77

7. Rozenfeld S. Drug adverse events in hospitals in the State of Rio de Janeiro, Brazil. Rev Saude Publ. 2007;41(1):108-15. http://dx.doi. org/10.1590/S0034-89102006005000012

8. Cox AR, Anton C, Goh CH, Easter M, Langford NJ, Ferner RE. Adverse drug reactions in patients admitted to hospital identified by discharge ICD-10 codes and by spontaneous reports. Br J Clin Pharmacol. 2001;52(6):337-9. http://dx.doi.org/10.1046/j.03065251.2001.01454.x 
9. Lugardon S, Desboeuf K, Fernet P, Montastruc JL, Lapeyre-Mestre M. Using a capture-recapture method to assess the frequency of adverse drug reactions in a French university hospital. Br J Clin Pharmacol. 2006;62(2):225-31. http://dx.doi.org/10.1111/j.13652125.2006.02633.x

10. Romero AV, Malone DC. Accuracy of adverse-drug-event reports collected using an automated dispensing system. Am J Health Syst Pharm. 2005;62(13):1375-80. http://dx.doi.org/10.2146/ ajhp040345

11. Otero MJ, Dominguez-Gil A. Drug-induced adverse avents. An emerging pa-thology. Farm Hosp. 2000;24:258-66.

12. Institute for Healthcare Improvement. Trigger tool for measuring adverse drug events [Internet]. Cambridge, MA: IHI; 2004. [cited 2015 Dez 07]. Available from: http://www.ihi.org/NR/ rdonlyres/8D970CE4-BF8C-4F35-9BC1-51358FC8B43F/2222/ TriggerToolforMeasuringAdverseDrugEventsCorrected1.pdf

13. Senst BL, Achusim LE, Genest RP, Cosentino LA, Ford CC, Little JA, Raybon SJ, Bates DW. Practical approach to determining costs and frequency of adverse drug events in a health care network. Am J Health Syst Pharm. 2001;58(12):1126-32.

14. Resar RK, Rozich JD, Classen D. Methodology and rationale for the measurement of harm with trigger tools. Qual Saf Health Care. 2003; 12 Suppl 2:ii39-45. http://dx.doi.org/10.1136/qhc.12.suppl_2.ii39

15. Forster AJ, Halil RB, Tierney MG. Pharmacist surveillance of adverse drug events. Am J Health Syst Pharm. 2004;61(14):1466-72.

16. Giordani F, Rozenfeld S, de Oliveira DF, da Silva Versa GL, Terencio JS, Caldeira Lde F, Gonçalves de Andrade LC. Vigilância de eventos adversos a medicamentos em hospitais: aplicação e desempenho de rastreadores. Rev Bras Epidemiol. 2012;15(3):455-67. http://dx.doi. org/10.1590/S1415-790X2012000300002

17. Handler SM, Altman RL, Perera S, Hanlon JT, Studenski SA, Bost JE, Saul MI, Fridsma DB. A systematic review of the performance characteristics of clinical event monitor signals used to detect adverse drug events in the hospital setting. J Am Med Inform Assoc. 2007;14(4):451-8. http://dx.doi.org/10.1197/jamia.M2369
18. Tribi-o G, Maldonado C, Segura O, Díaz J. Costos directos y aspectos clínicos de las reacciones adversas a medicamentos en pacientes hospitalizados en el servicio de medicina interna de una instituición de tercer nivel de Bogotá. Biomedica. 2006;26(1):31-41. http:// dx.doi.org/10.7705/biomedica.v26i1.1392

19. Schade CP, Hannah K, Ruddick P, Starling C, Brehm J. Improving self-reporting of adverse drug events in a West Virginia hospital. Am J Med Qual. 2006;21(5):335-41. http://dx.doi.org/10.1177/ 1062860606291322

20. Weingart SN, Toth M, Eneman J, Aronson MD, Sands DZ, Ship AN, Davis RB, Phillips RS. Lessons from a patient partnership intervention to prevent adverse drug events. Int J Qual Health Care. 2004;16(6):499-507. http://dx.doi.org/10.1093/intqhc/ mzh083

21. Davies EC, Green CF, Mottram DR, Pirmohamed M. Adverse drug reactions in hospital in-patients: a pilot study. J Clin Pharm Ther. 2006;31(4):335-41. http://dx.doi.org/10.1111/j.13652710.2006.00744.x

22. Hwang SH, Lee S, Koo HK, Kim Y. Evaluation of a computerbased adverse-drug-event monitor. Am J Health Syst Pharm. 2008;65(23):2265-72. http://dx.doi.org/10.2146/ajhp080122

23. Silva DC, Araujo OR, Arduini RG, Alonso CF, Shibata AR, Troster EJ. Adverse drug events in a pediatric intensive care unit: a prospective cohort. BMJ Open. 2013;3(2).pii:e001868. http:// dx.doi.org/10.1136/bmjopen-2012-001868

24. Classen DC, Pestotnik SL, Evans RS, Burke JP. Computerized surveillance of adverse drug events in hospital patients. JAMA. 1991;266(20):2847-51. http://dx.doi.org/10.1001/jama.1991. 03470200059035

25. Sánchez Mu-oz-Torrero JF, Barquilla P, Velasco R, Fernández Capitan Mdel C, Pacheco N, Vicente L, Chicón JL, Trejo S, Zamorano J, Lorenzo Hernandez A. Adverse drug reactions in internal medicine units and associated risk factors. Eur J Clin Pharmacol. 2010;66(12):1257-64. http://dx.doi.org/10.1007/s00228-0100866-6 